\title{
Experimental ocular siderosis after extrabulbar administration of iron
}

\author{
K GERKOWICZ, M PROST, AND M WAWRZYNIAK \\ From the Clinic of Ophthalmology, Medical Academy in Lublin, Poland
}

SUMMARY The authors studied the penetration of iron administered extrabulbarly into the ocular tissues of rabbits. It was found that iron passes from the orbit into the eyeball and accumulates in considerable quantities in the sclera, choroid, retina, ciliary body, and even in the vitreous and corneal epithelium. However, light microscopy failed to show any damage to the ocular tissues. The mechanism by which iron penetrates into the eyeball is discussed, and comparison is made between changes in the tissues, which characterise siderosis produced by an intrabulbar iron foreign body, and those in which an extrabulbar foreign body is involved.

Changes which are produced by intrabulbar iron splinters are well known to every ophthalmologist, but there is less knowledge of changes caused by iron that has penetrated into the orbit in the vicinity of the eyeball. Most clinical reports point to the absence of any harmful action of such foreign bodies.' There is, however, a report of a case of siderosis cataract produced by an orbital foreign body adhering to the eyeball. ${ }^{2}$ The objective of the present study was to find out to what extent, if at all, iron can penetrate from the orbit into the eyeball, and especially into the retina.

\section{Material and methods}

Twenty four eyes of 12 albino rabbits weighing 2.5-3 $\mathrm{kg}$ were used for the study. In each animal the bulbar conjunctiva of both eyes was incised in the inferonasal quadrant, and after exposing the sclera $15 \mathrm{mg}$ of iron in powder form was placed in the equator region. The conjunctiva was closed with a continuous suture, which was removed on the seventh day. In the fifth, ninth, and twelfth month after the administration of iron the eyeballs were taken for histological examination after systemic perfusion of the blood vessels to prevent distortion of tissues in the fixation process. The perfusion was carried out as follows: after exposing the heart we introduced a metal cannula into the ascending aorta through the left heart ventricle. The cannula was connected to a transfusion

Correspondence to Professor K Gerkowicz, Clinic of Ophthalmology, Chmiclna 1, 20-079 Lublin, Poland. apparatus with $1000 \mathrm{ml}$ of a perfusion liquid containing $2 \%$ paraformaldehyde and $2 \cdot 5 \%$ glutaraldehyde. The liquid was introduced at a pressure of $90-100$ $\mathrm{mmHg}$. The eyeballs were collected $15 \mathrm{~min}$ after the cessation of the perfusion and were fixed according to the Szent-Györgyi technique. Histological sections were stained with haematoxylin and eosin. The Prussian blue reaction to $\mathrm{Fe}^{3+}$ and the Turnbull blue reaction to $\mathrm{Fe}^{2+}$ were also applied. ${ }^{3}$ Both methods of detecting iron were modified to eliminate staining of

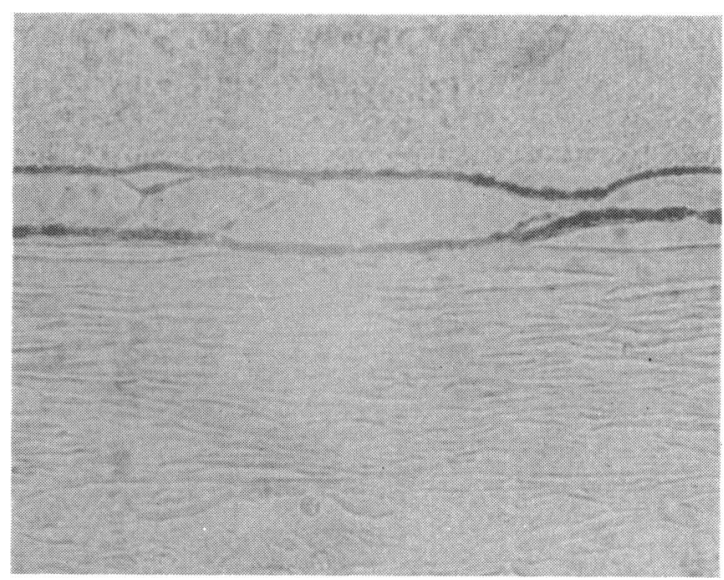

Fig. 1 The sclera, choroid, and retina of a rabbit in which no iron was found. There is no staining of the individual morphological elements. The darker stain of the pigment epithelium and of part of the choroid is due to the presence of natural pigments. (Prussian blue reaction, $\times 150)$. 
the background (nuclei and cytoplasm), so that the blue staining appeared only in those parts of the tissues which contained iron. For this reason the preparations of tissues free from iron remained unstained (Fig. 1). Since only slight changes were found in the eyes of the two rabbits killed in the fifth month, the eyeballs of the remaining 10 rabbits were collected in the ninth and twelfth months, from five animals at a time.

\section{Results}

The results of histological examination showed that iron placed in the orbit near the sclera penetrated inside the eyeball, and that it was a very slow process. Five months after the administration of iron its deposition could be observed only in the superficial layers of the sclera, so that not more than one third of the thickness of the tissue was involved (Fig. 2). Small amounts of iron were also found in the subconjunctival tissue away from the site of the introduction of the iron powder. This penetration of iron seemed to favour the anterior direction, and traces of the metal were also observed under the conjunctiva in the limbus. In other parts of the eye no evidence of the presence of iron could be found.

After nine and 12 months the penetration of iron into the eyeball was much more pronounced. In these experimental groups the histological findings were similar, though the penetration of iron into the retina, choroid, and ciliary body was usually slightly stronger after 12 months than after nine months. However, these differences were by no means significant. At the same time considerable differences in

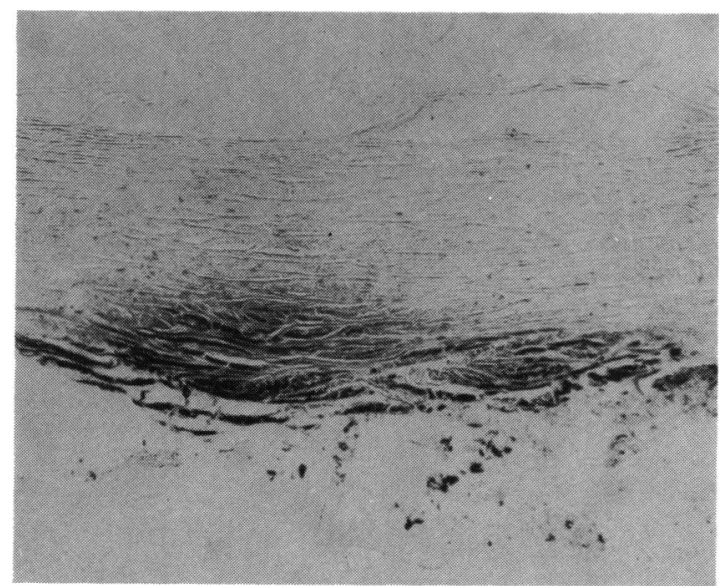

Fig. 2 Histological changes in the sclera five months after administration of iron. There is diffuse staining of the external one-third of the tissue. (Prussian blue reaction, $\times 112$ ).

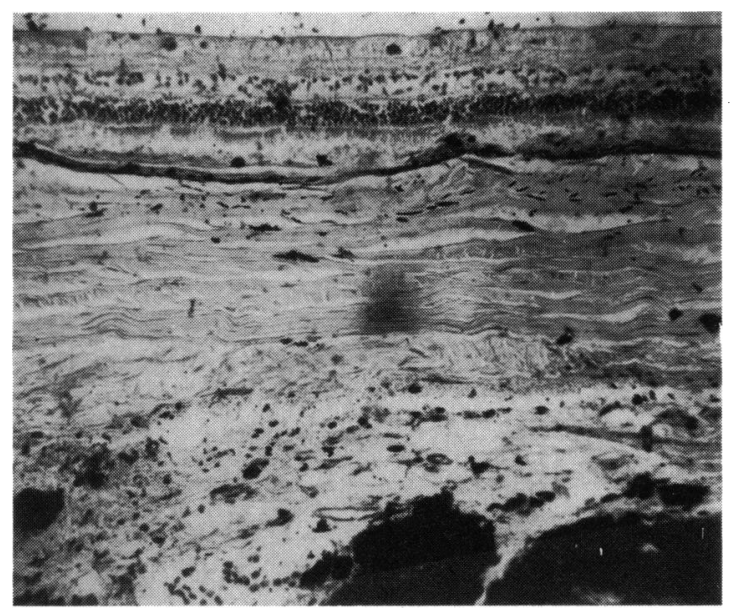

Fig. 3 Histological appearance of the retina, choroid, and sclera 12 months after administration of iron. There is diffuse and granular staining of all layers of the eyeball wall. (Prussian blue reaction, $\times 150$ ).

the penetration process were seen in the individual animals. For these reasons, and because it was not possible to distinguish changes characteristic of either time period, both experimental groups are discussed jointly.

Preparations stained with the use of the reactions to $\mathrm{Fe}^{2+}$ and $\mathrm{Fe}^{3+}$ showed that iron present in the tissues had produced two forms of staining: either minute and strongly stained granular agglomerations, or diffuse staining of whole tissues or of their parts. In the vicinity of the deposited iron powder the penetration of the metal through the whole sclera appeared in the form of both diffuse and granular staining (Fig. 3). In the parts of the sclera furthest away from the implantation site changes were observed in the external layers of the tissue, and the staining was diffuse. As far as the choroid is concerned, iron could be seen primarily in the vessel walls. The site of an especially intensive accumulation of iron was the pigment epithelium of the retina (Fig. 3). Bruch's membrane took a weak stain and could hardly be seen in the preparations (Fig. 4).

Iron usually pervaded the whole retina. This appeared as diffuse staining of all its layers. The most intensive staining was present in the external and internal nuclear layer (Fig. 3). The neuroepithelium and the nerve fibre layer showed a weaker stain, but the walls of the retinal vessels and the internal limiting membrane stained fairly strongly (Fig. 4). Strongly stained agglomerations of iron were also seen scattered in all layers of the retina-on its surface, and in the adjacent part of the vitreous (Fig. 4). Occasionally a detachment of the vitreous was observed. Its surface showed a fairly strong staining. 


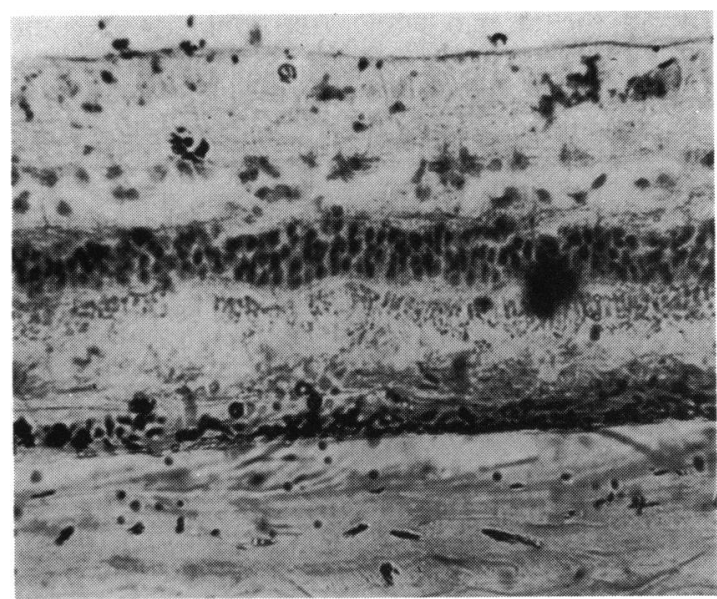

Fig. 4 Histological changes in the retina 12 months after administration of iron. There is staining of all layers of the retina and especially strong reaction in the pigment epithelium, internal and external nuclear layer, and internal limiting membrane. There is staining of iron agglomerations. (Turnbull blue reaction, $\times 264$ ).

Of the other parts of the eye, the ciliary body, and above all its processes, were observed to have accumulated iron, mostly in the ciliary epithelium. The stroma of the ciliary body showed a less intense staining but contained agglomerations of iron (Fig. 5). Small traces of iron were found in the iris and anterior chamber angle. A diffuse staining was present in the corneal epithelium, but in other parts of the cornea iron could not be detected.

The blue stain owing to the presence of iron,

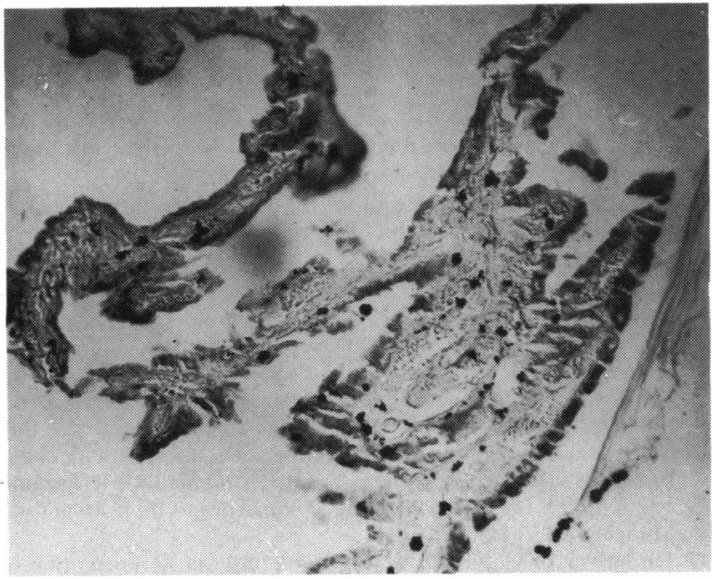

Fig. 5 Histological appearance of the ciliary processes, 12 months after administration of iron. There is strong diffuse staining of the ciliary epithelium and less intense staining of the stroma, in which iron agglomerations are visible.

(Prussian blue reaction, $\times 264)$. was more intense in the $\mathrm{Fe}^{3+}$ reaction than in the $\mathrm{Fe}^{2+}$ reaction. In preparations stained with haematoxylin and eosin no distinct changes were found that could point to damage sustained by the ocular tissues. The only change was a spreading apart of the collagen fibres of the sclera in the sites of iron accumulation.

\section{Discussion}

The results of our study indicate that iron, when administered extrabulbarly, penetrates to a large extent into the eyeball and accumulates in the ocular tissues, among others in the retina. Two forms of iron staining were seen in the tissues: the diffuse and the granular form. It seems that the diffuse stain results from the penetration of iron through successive layers of the eyeball wall, while the granular form of iron is probably transported to the tissues by migrating cells.

The histological changes observed in this experiment are considerably different from those in siderosis caused by an intrabulbar foreign body. In the latter case iron is deposited above all in the ciliary epithelium, iris, and pigment epithelium of the retina, and in the internal layers of the retina in man and monkeys, ${ }^{4-7}$ or in the external layers of the retina of the rabbit. ${ }^{47}$ Smaller amounts of iron were found in the epithelium and fibres of the lens and in the epithelium, stroma, and endothelium of the cornea ${ }^{48}$ No accumulation of the metal could be found in the choroid or sclera. ${ }^{457}$ The changes were accompanied by extensive destruction and atrophy of the retina, while there was basically no damage to the iris, ciliary body, or cornea.$^{4}$

Two fundamental differences in the histological picture were found by us after extrabulbar administration of iron, as compared with the changes observed by other authors with regard to intrabulbar iron foreign bodies. First, iron accumulated in the sclera, choroid, pigment epithelium of the retina, epithelium and stroma of the ciliary body, and in the corneal epithelium. However, no deposition of iron was detected in the iris or in the stroma and endothelium of the cornea. Second, there was no visible damage to the retina, as evidenced by preparations stained with haematoxylin and eosin. The above differences were probably linked with the rate at which, and the route by which, iron penetrated into the individual ocular tissues. The presence of an intrabulbar foreign body results in a massive and violent release of iron ions into the vitreous and from there into the retina. Some of the ions are bound in the tissues by iron binding substances, for example, apoferritin. It is thought that the remaining free ions, having undergone a redox reaction with the participation of ascorbic acid, contribute to the formation of 
free radicals $\mathrm{OH}^{\cdot}$ and $\mathrm{OH}_{2}{ }^{-}$- These radicals depolymerise hyaluronic acid of the vitreous, reduce the content of non-saturated fatty acids, and oxidise lipids to lipoperóxides in the retina. ${ }^{911} 12$ This results in damage to the retina because of inactivation of enzymes containing sulphhydryl groups, and in disintegration of amino acids and proteins. It was also found that lipoperoxides reduce the respiratory function of the retina and inhibit the regeneration of rhodopsin." Iron ions also form stable complexes with sulphhydryl groups and imidazole proteins, which again results in inactivation of enzymes. ${ }^{13}$

Of the large quantity of iron ions released from an intrabulbar foreign body, only a small percentage can be removed by iron binding systems, for example, by ferritin, in the retina. The remaining free iron ions, acting through the processes described above, cause considerable damage to the retina.

A different situation arises with an iron foreign body situated outside the eyeball. The penetration of iron ions into the retina is very slow, and only a small number of ions can invade the eye at a given moment. It also seems that not all iron ions that have crossed the sclera reach the retina. Some of them can be conveyed to the anterior chamber via the suprachoroidal space. This supposition is strengthened by the finding that iron easily penetrates from the anterior chamber into the suprachoroidal space through the anterior chamber angle, even in initial stages of siderosis. ${ }^{8}$ Bruch's membrane is probably another barrier to the penetration of iron. ${ }^{15}$ Apart from that, a quantity of the metal can be bound in the sclera and choroid. All these factors may have contributed to the observation that only a small amount of iron, and at a slow rate, was able to infiltrate the retina. In this situation the iron binding properties of the retina could prove adequate to prevent the appearance of histologically detectable changes. However, the question remains open whether constant infiltration of iron for a period longer than 12 months will still be successfully counteracted by this defence system of the retina.

The penetration of iron from the orbit into the eyeball has recently been the subject of other experimental studies. Sinovich and Gudkova ${ }^{14}$ found accumulation of iron in the retina and choroid of rats but failed to observe morphological changes in these tissues. Our previous work ${ }^{15}$ showed that the presence of an iron foreign body in the orbit results in a considerable increase of iron concentration in the aqueous of rabbits. Burch and Albert' implanted iron splinters into the orbit and sclera of rabbits and observed an accumulation of the metal mainly in the sclera, choroid, and ciliary body, and only occasionally in the retina. On the other hand they frequently found local atrophy of the retina and choroid in the site of the foreign body. According to the same authors the typical site of the accumulation of iron that has penetrated from the orbit are the tissues situated at the junction of the sclera and choroid. In our study accumulation of iron was also seen in the retina, and even in the vitreous, in almost every animal. However, it should be borne in mind that our application of iron in the form of powder may have facilitated the penetration of the metal into the eyeball. Interestingly enough, we did not observe particularly strong reactions to iron in the tissues situated at the boundary between the sclera and choroid. It seems that such changes can be a transitional stage during the migration of iron into the eye. Since the penetration of iron in our experiment was faster than that observed by Burch and Albert, the infiltration of the metal at the junction of the sclera and choroid might have occurred between the fifth and ninth months of the experiment and therefore might have remained unnoticed by us.

The results of the present study speak in favour of the removal of iron splinters from the orbit, as it can be supposed that constant penetration of iron for a period longer than that observed in our experiment can lead to siderosis of the retina and, by that, to its damage.

\section{References}

1 Burch PG, Albert DM. Transscleral ocular siderosis. Am J Ophthalmol 1977; 84: 90-7.

2 Sironi L. Siderotic cataract due to metallic splinter adhering to lateral wall of orbit without penetrating eyeball. Boll Oculist 1942; $21: 585$

3 Gabe M. Histological techniques. Paris: Masson, 1976.

4 Declercq SS, Meredith PCA, Rosenthal AR. Experimental siderosis in the rabbit. Arch Ophthalmol 1977; 95: 1051-8.

5 Masciulli L, Anderson DR, Charles S. Experimental ocular siderosis in the squirrel monkey. Am J Ophthalmol 1972; 74: 638-61.

6 Vogel M. Morphologie der Metallosen. In: Neubauer $\mathbf{H}$, Rüssmann W, Kilp $\mathrm{H}$, eds. Intraokulare Fremdkörper und Metallose. München: Bergmann, 1977: 9-15.

7 Wise JB. Treatment of experimental siderosis bulbi, vitreous hemorrhage and corneal bloodstaining with deferoxamin. Arch Ophthalmol 1966; 75: 698-707.

8 Appel J, Barishak YR. Histopathological changes in siderosis bulbi. Ophthalmologica 1978; 176: 205-10.

9 Hofmann H, Khan MAH, Schmut O. Der Einfluss von Katalase und Superperoxydoismutase auf dic Viskositätsänderung von Hyaluronsäurelösungen aus Rinderglaskörpern nach Zusatz von Eisenionen. Klin Monatsbl Augenheilkd 1983; 182: 214-7.

10 Schmut O. Hofmann H. Verflüssigung des Glaskörpers/Mechanismus der Glaskörperverflüssigung. In: Ncubaucr $\mathrm{H}$, Rüssmann W, Kilp H, cds. Intraokulare Fremdkörper und Metallose. München: Bergmann, 1977: 73-7.

11 Hiramitsu T, Majima T, Hascgawa Y, Hirata K. Formation of lipoperoxide in the retina in ocular siderosis. In: Ncubaucr $\mathbf{H}$, Rüssmann W, Kilp H, cds. Intraokulare Fremdkörper und Metallose. München: Bergmann, 1977: 89-92.

12 Weiss H, Graf A. Über die Schädigung des retinalen Fettsäurenmusters nach intravitrealer Eiseninjektion. Albrecht von Graefes Arch Klin Ophthalmol 1975; 197: 293-8. 
13 Rüssmann W. Biochemie der Metallosen. In: Neubauer $\mathrm{H}$, Rüssmann W, Kilp H, eds. Intraokulare Fremdkörper und Metallose. München: Bergmann, 1977: 45-53.

14 Sinovich VA, Gudkova EV. The effect of foreign metal bodies in the orbit on the cycball coats. Vestn Oftalmol 1969; 82: 10-3.

15 Gerkowicz K. Prost M. Experimental investigations on the penetration into the cycball of iron administered intraorbitally. Ophthalmologica 1984: 188 : 239-42. 